BULLETIN OF THE

AMERICAN MATHEMATICAL SOCIETY

Volume 80, Number 3, May 1974

\title{
A PHYSiCAlly CONSISTENT, DISCRETE $n$-BODY MODEL
}

\author{
BY DONALD GREENSPAN
}

Communicated by Fred Brauer, October 8, 1973

1. Introduction. A dynamical model of a physical phenomenon is said to be physically consistent if the model (a) conserves energy, (b) conserves linear momentum, (c) conserves angular momentum, and (d) is symmetric in the physical sense, that is (see [3]), the form of its dynamical equations is invariant with respect to translation, rotation and uniform motion. Unfortunately, the equations of many interesting, physically consistent models have eluded solution by analytical mathematical methods. It was natural then, with the emergence of the modern digital computer, that there were developed varieties of discrete $n$-body, "molecular" type, models which were used to approximate solutions of such equations (see, e.g., [1], [2], [4], [7]-[12], and the additional references contained therein). However, none of these discrete models was, itself, physically consistent, which forced the unnatural development of computational contrivances, like "artificial viscosity", in order to achieve numerical stability.

Our objective here, then, is to construct a physically consistent, discrete $n$-body model. Our approach will be of sufficient generality so as to include both Newtonian and classical molecular $n$-body forces. During the discussion, it will be worth noting that, unlike other discrete approaches, our definitions of kinetic energy, potential energy, linear momentum, and angular momentum are identical with those of classical, continuous $n$-body models.

2. The discrete model. For a positive time step $\Delta t$, let $t_{k}=k \Delta t$, $k=0,1,2, \cdots$. At time $t_{k}$, let particle $P_{i}$ of mass $m_{i}$ be located at $\boldsymbol{x}_{i, k}=\left(x_{i, k}, y_{i, k}, z_{i, k}\right)$, have velocity $\boldsymbol{v}_{i, k}=\left(v_{i, k, x}, v_{i, k, y}, v_{i, k, z}\right)$, and have acceleration $a_{i, k}=\left(a_{i, k, x}, a_{i, k, y}, a_{i, k, z}\right)$, for $i=1,2, \cdots, n$. Position, velocity, and acceleration are assumed to be related by the formulas

$$
\begin{gathered}
\left(v_{i, k+1}+v_{i, k}\right) / 2=\left(x_{i, k+1}-x_{i, k}\right) /(\Delta t), \\
a_{i, k}=\left(v_{i, k+1}-v_{i, k}\right) /(\Delta t) .
\end{gathered}
$$

If $F_{i, k}=\left(F_{i, k, x}, F_{i, k, y}, F_{i, k, z}\right)$ is the force acting on $P_{i}$ at time $t_{k}$, then force and acceleration are assumed to be related by the discrete dynamical

AMS (MOS) subject classifications (1970). Primary 65L05, 70F10. 
equation

$$
\boldsymbol{F}_{i, k}=m_{i} \boldsymbol{a}_{i, k} .
$$

In particular, we will consider only forces which have components of attraction which behave like $p /\left(r^{\alpha}\right)$ and components of repulsion which behave like $q /\left(r^{\beta}\right)$, where $p, q, \alpha, \beta$ are nonnegative parameters with $\beta>\alpha \geqq 2$, and where $r$ is the distance between a pair of particles, as follows [5]. Let $r_{i j, k}$ be the distance between $P_{i}$ and $P_{j}$ at $t_{k}$. Then $F_{i, k}$, the force exerted on $P_{i}$ at time $t_{k}$ by the remaining $(n-1)$ particles $P_{j}$ of the system, is defined by

$$
\begin{aligned}
\boldsymbol{F}_{i, k}=m_{i} \sum_{j=1 ; j \neq i}^{n}\left\{m_{j}(\right. & -\frac{p \sum_{\xi=0}^{\alpha-2}\left(r_{i j, k}^{\xi} r_{i j, k+1}^{\alpha-\xi-2}\right)}{r_{i j, k}^{\alpha-1} r_{i j, k+1}^{\alpha-1}\left(r_{i j, k+1}+r_{i j, k}\right)} \\
\left.+\frac{q \sum_{\xi=0}^{\beta-2}\left(r_{i j, k}^{\xi} r_{i j, k+1}^{\beta-\xi-2}\right)}{r_{i j, k}^{\beta-1} r_{i j, k+1}^{\beta-1}\left(r_{i j, k+1}+r_{i j, k}\right)}\right) & \left.\cdot\left(x_{i, k+1}+x_{i, k}-x_{j, k+1}-x_{j, k}\right)\right\}
\end{aligned}
$$

Finally, at $t_{k}$, the kinetic energy $K_{k}$, potential energy $V_{k}$, linear momentum $\boldsymbol{M}_{k}$, and angular momentum $\boldsymbol{L}_{k}$ of the system are defined by

$$
\begin{gathered}
K_{k}=\sum_{i=1}^{n} \frac{1}{2} m_{i}\left(v_{i, k, x}^{2}+v_{i, k, y}^{2}+v_{i, k, z}^{2}\right), \\
V_{k}=\sum_{i, j=1 ; i<j}^{n}\left(\frac{-p}{r_{i j, k}^{\alpha-1}}+\frac{q}{r_{i j . k}^{\beta-1}}\right) m_{i} m_{j}, \\
\boldsymbol{M}_{k}=\sum_{i=1}^{n} m_{i} \boldsymbol{v}_{i, k}, \\
\boldsymbol{L}_{k}=\sum_{i=1}^{n} m_{i}\left(\boldsymbol{x}_{i, k} \times \boldsymbol{v}_{i, k}\right) .
\end{gathered}
$$

3. Physical consistency of the discrete model. We will show now that the discrete model of $\$ 2$ satisfies the physical consistency criteria (a)-(d) of $\S 1$.

(a) Conservation of energy. For the proof, see [5].

(b) Conservation of linear momentum. From (2.3) and (2.4), note that

$$
\sum_{i=1}^{n} m_{i} a_{i, k}=0, \quad k=0,1,2, \cdots .
$$

From (2.2) and (3.1), then,

$$
\sum_{i=1}^{n} m_{i} \boldsymbol{v}_{i, k}=\sum_{i=1}^{n} m_{i} \boldsymbol{v}_{i, 0}, \quad k=1,2, \cdots
$$


From (2.7) and (3.2), finally,

$$
\boldsymbol{M}_{k}=\sum_{i=1}^{n} m_{i} \boldsymbol{v}_{i, 0}, \quad k=1,2, \cdots
$$

(c) Conservation of angular momentum. From (2.1)-(2.3) and (2.8),

$$
\boldsymbol{L}_{k+1}-\boldsymbol{L}_{k}=\Delta t \sum_{i=1}^{n}\left(\frac{\boldsymbol{x}_{i, k+1}+\boldsymbol{x}_{i, k}}{2}\right) \times \boldsymbol{F}_{i, k}
$$

Let the torque $\boldsymbol{T}_{k}$ of the system at $t_{k}$ be defined by

$$
\boldsymbol{T}_{k}=\sum_{i=1}^{n}\left(\frac{\boldsymbol{x}_{i, k+1}+\boldsymbol{x}_{i, k}}{2}\right) \times \boldsymbol{F}_{i, k} .
$$

Then, $\boldsymbol{T}_{k} \equiv \mathbf{0}$ implies $\boldsymbol{L}_{k}=\boldsymbol{L}_{0}, k=1,2, \cdots$.

(d) Symmetry. For the proof, see [6].

\section{REFERENCES}

1. G. Birkhoff and R. E. Lynch, Lagrangian hydrodynamic computations and molecular models of matter, LA-2618, Los Alamos Sci. Lab., Los Alamos, N.M., 1961.

2. B. J. Daly, F. H. Harlow and J. E. Welch, Numerical fluid dynamics using the particle-and-force method, LA-3144, Part I, Los Alamos Sci. Lab., Los Alamos, N.M., 1965.

3. R. P. Feynman, R. B. Leighton and M. Sands, The Feynman lectures on physics, Vol. I: Mainly mechanics, radiation and heat, Addison-Wesley, Reading, Mass., 1963. MR 35 \#3942.

4. J. B. Gibson, A. N. Goland, M. Milgram and G. H. Vineyard, Dynamics of radiation damage, Phys. Rev. 120 (1960), 1229-1253.

5. D. Greenspan, An algebraic, energy conserving formulation of classical molecular and Newtonian n-body interaction, Bull. Amer. Math. Soc. 79 (1973), 423-427.

6. - Symmetry in discrete mechanics, Found. Phys. 3 (1973), 247-253.

7. A. B. Langdon, 'Energy-conserving' plasma simulation algorithms, J. Computational Phys. 12 (1973), 247-268.

8. A. K. MacPherson, The formation of shock waves in a dense gas using a molecular dynamics type technique, J. Fluid Mech. 45 (1971), 601-621.

9. J. von Neumann, Proposal and analysis of a new numerical method for the treatment of hydrodynamical shock problems, Collected works. Vol. VI: Theory of games, astrophysics, hydrodynamics and meteorology, A Pergamon Press Book, Macmillan, New York, 1963. MR 28 \#1105.

10. J. R. Pasta and S. Ulam, Heuristic numerical work in some problems of hydrodynamics, Math. Tables Aids Comput. 13 (1959), 1-12. MR 21 \#2348.

11. Yu. P. Popov and A. A. Samarskili, Completely conservative difference schemes for magnetohydrodynamic equations, U.S.S.R. Comput. Math. and Math. Phys. 10 (1970) 233-243.

12. L. Verlet, Computer experiments on classical fluids. II. Equilibrium correlation functions, Phys. Rev. 165 (1968), 201-214.

Department of Computer Sciences, University of Wisconsin, Madison, WISCONSIN 53706 